\title{
Linking Ambulance Records with Hospital and Death Index Data to Evaluate Patient Outcomes
}

\author{
Emily Andrew ${ }^{1,2, *}$ \\ Shelley Cox ${ }^{1,2, *}$ \\ Karen Smith ${ }^{1-3}$
}

'Centre for Research and Evaluation, Ambulance Victoria, Victoria, Australia; ${ }^{2}$ Department of Epidemiology and Preventive Medicine, Monash University, Victoria, Australia; ${ }^{3}$ Department of Paramedicine, Monash University, Victoria, Australia

*These authors contributed equally to this work
Correspondence: Emily Andrew

Email emily.andrew@ambulance.vic.gov.au
Objective: Linkage of electronic administrative datasets is becoming increasingly common, offering a powerful resource for research and analysis. However, routine linkage of prehospital data with emergency department (ED) presentation and hospital admission datasets is rare. We describe a methodology used to link ambulance data with hospital ED presentations, admissions, and death records, and examine potential biases between matched and unmatched patients.

Methods: Iterative deterministic linkage methodologies were employed to link clinical, operational, and secondary triage ambulance data to ED presentations, hospital admissions, and death records in Victoria, Australia. Descriptive analyses and standardised differences were used to examine potential biases between matched and unmatched patients.

Results: A total of 2,813,913 ambulance records were available for linkage. Of the patients that were transported to a public ED ( $\mathrm{n}=1,753,268), 83.3 \%$ matched with an ED record. Only small differences were observed between matched and unmatched patients for sex, year, time of day and attending crew type. The data elements with the largest standardised differences were patient age $(0.25)$ and paramedic diagnosis $(0.25)$. Matched patients were older (mean \pm standard deviation: $55.6 \pm 25.7$ vs $49.0 \pm 26.0$ years) and more likely to have a paramedicsuspected cardiac, respiratory, neurological, or gastrointestinal/genitourinary condition, suspected infection/sepsis, or pain.

Conclusion: This linked dataset will facilitate a large body of research into prehospital care and patient outcomes. Although future analysis of matched patients should acknowledge the linkage error rate, our findings suggest that results are likely to be generalisable to the broader ambulance population.

Keywords: ambulance, prehospital, data linkage, outcomes

\section{Introduction}

Linkage of electronic administrative datasets is becoming increasingly common, offering a powerful resource for research and analysis. ${ }^{1}$ In the prehospital setting, completion of electronic patient care records at the conclusion of each case is becoming common place, the data from which are stored in data warehouses. However, routine linkage of this data with emergency department (ED) presentation and hospital admission datasets is rare and has historically been performed on a project-by-project basis. ${ }^{2-7}$ Consequently, the influence of prehospital triage and care on patient outcomes is not systematically evaluated by ambulance services internationally.

This paper describes the methodology used in Victoria, Australia to link ambulance data with hospital ED presentations, admissions, and death records. It also examines potential biases between matched and unmatched patients to understand 
whether the cohort of matched patients is representative of all ambulance callers. This linked dataset will facilitate a large body of research into the epidemiology of patients accessing ambulance care, as well as the impact of paramedic diagnosis, treatment, and triage on patient outcomes. The data will also serve as a valuable resource for validating call-taker dispatch algorithms and the associated ambulance response.

\section{Methods}

\section{Setting}

Ambulance Victoria is the single ambulance service in Victoria, serving a population of $>6.5$ million people. Calls for ambulance are triaged using the Medical Priority Dispatch System which determines the caller's primary complaint and is linked with a pre-determined level of ambulance response. The response may include dispatch of an emergency ambulance or referral to the Ambulance Victoria Secondary Triage Service for additional questioning by an experienced clinician. This secondary triage may result in the dispatch of an emergency ambulance, non-emergency patient transport vehicle, referral to an alternative health service, or provision of self-care advice.

\section{Data Sources}

\section{Ambulance Data Sources}

Operational (eg Computer Aided Dispatch), electronic patient care record and secondary triage data relating to consecutive emergency ambulance calls between 1 January 2015 and 30 June 2019 were extracted from the Ambulance Victoria data warehouse. The warehouse stores these three datasets in separate subject areas. Accordingly, iterative deterministic linkage was used to link records based on an ambulance "common event number", case date, case number and patient identifiers (name, date of birth, residential postcode/suburb).

\section{State-Wide Registry Data}

Data from two state-wide clinical quality registries were included in the ambulance dataset:

1. The Victorian Ambulance Cardiac Arrest Registry, maintained by Ambulance Victoria, captures Utstein-style data related to all out-of-hospital cardiac arrests attended by ambulance in the state. ${ }^{8}$

2. The Victorian State Trauma Registry routinely provides Ambulance Victoria with a subset of data related to all major trauma patients from every hospital and healthcare facility in Victoria. ${ }^{9}$

Iterative deterministic linkage based on ambulance case date, case number and patient identifiers (name, date of birth, residential postcode) was used to link ambulance and registry data.

\section{Data Linkage with Hospital and Death Index Data}

Ambulance data were provided to the Centre for Victorian Data Linkage, housed within the Victorian Department of Health, for linkage with three administrative datasets:

1. Victorian Emergency Minimum Dataset (VEMD) which contains administrative and clinical data related to ED presentations at 39 public hospitals in Victoria. These 39 hospitals receive $>95 \%$ of all ambulance transports to public hospitals in the state.

2. Victorian Admitted Episodes Dataset (VAED) which captures demographic, clinical and administrative data relating to each admitted episode of care occurring in public and private hospitals, as well as rehabilitation centres, extended care facilities and day procedure centres.

3. Victorian Death Index (VDI) which captures the date and cause of all deaths registered in Victoria.

Deterministic linkage based on patient identifiers (name, date of birth, sex, residential postcode) was performed to match ambulance patients with patients present in the administrative datasets. Fuzzy matching was allowed on patient names. Unique patients were assigned an identification number and identifiers were stripped from the data. Content data, which is stored separately to identifiable data by the Centre for Victorian Data Linkage, were then extracted from the three administrative datasets named above for matched patients.

The matched patient-level data were then used by Ambulance Victoria to link episodes of care. For ambulance patients who were transported to hospital, ED presentations were linked to ambulance records where the VEMD arrival time was within two hours of the ambulance ED arrival time. To understand the outcomes of non-transported ambulance patients, ambulance records were linked to ED presentations occurring within 24,48 and 72 hours of the emergency call. If multiple VEMD records existed within the respective time period, the ED presentation occurring closest in time to the ambulance call was kept. Hospital admissions occurring 
within 24, 48 and 72 hours of the emergency ambulance call were also linked to the ambulance data. Where multiple admitted episodes were recorded within the respective time period, the episode occurring closest in time to the emergency ambulance call was kept. Death records were linked to all ambulance records for each patient within the study period.

\section{Statistical Analyses}

To understand whether systematic biases existed between matched and unmatched patients, we examined patients transported by ambulance to one of the 39 public EDs that report to the VEMD. This patient subgroup was analysed as a surrogate for the entire dataset as most patients were expected to have a matched VEMD record. We used descriptive statistics to compare matched and unmatched patients. Data are presented as proportions for categorical data and means (standard deviations) for continuous data. Due to the large sample size, standardised differences were used rather than p-values to measure the effect size between groups. Standardised differences of $0.2,0.5$ and 0.8 represent small, moderate, and large differences, respectively. ${ }^{10}$

\section{Ethics}

This project has ethical approval from the Monash University Human Research Ethics Committee (project \#11681). Patient consent was not required by the Ethics Committee as it was considered impractical to obtain consent from $>2.5$ million patients. Further, the Ambulance Victoria Privacy Policy and Victorian Department of Health guidelines state that data may be used for research purposes. As such, a waiver of consent was granted for this research. To ensure patient confidentiality, all data transfer was undertaken via secure data exchange. Patient identifiers were removed from the data following linkage such that researchers could only access de-identified data. This research was conducted in accordance with the Declaration of Helsinki.

\section{Results}

A total of 2,857,760 emergency calls were recorded during the 4.5-year study period. After excluding patients with missing identifiers (therefore precluding linkage with administrative datasets) and those who died at scene, 2,813,913 records were available for linkage (Figure 1). A total of $1,753,268$ patients were transported by

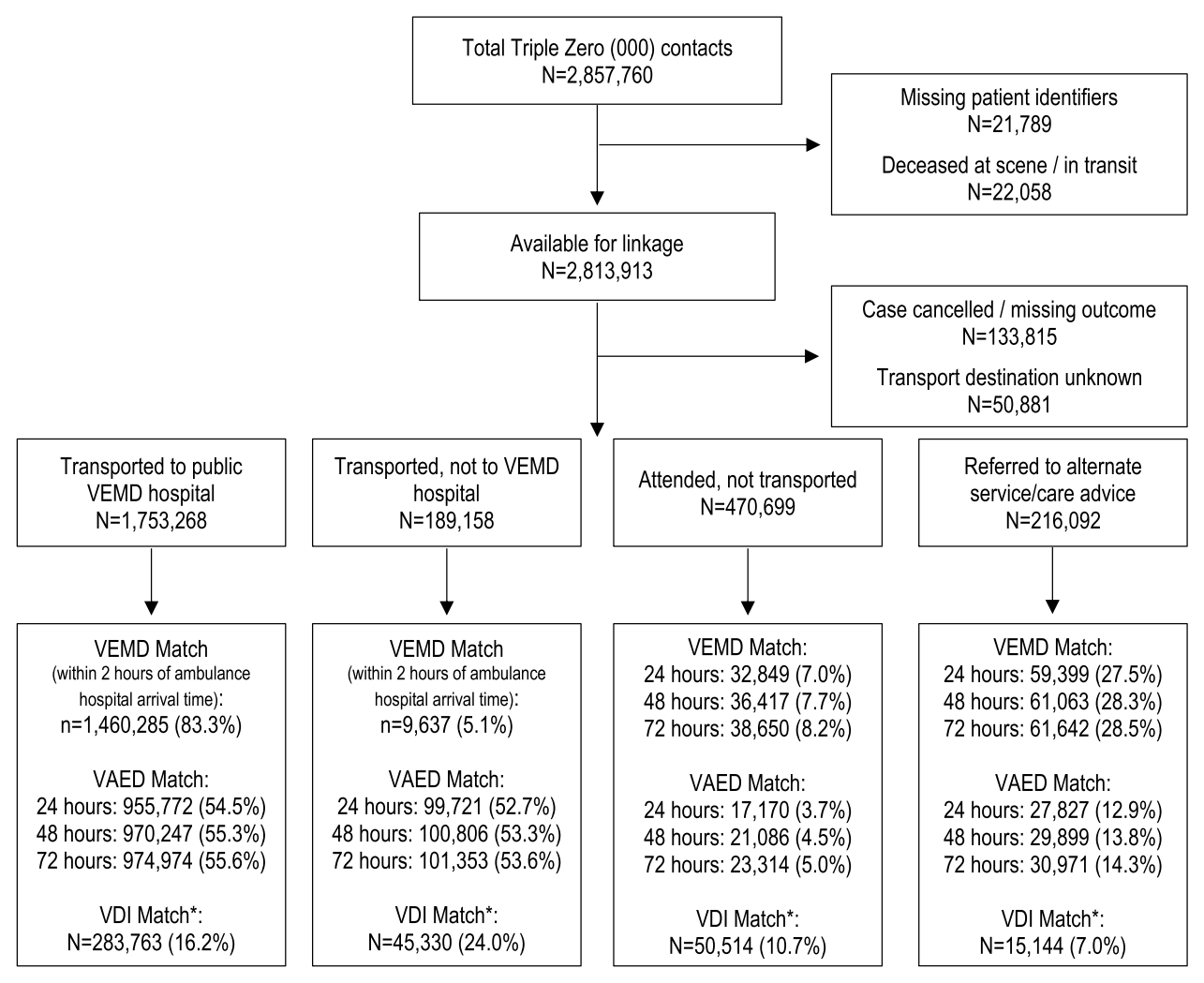

Figure I Linkage Flow Chart.

Note: *Date of death was matched to all ambulance records associated with a patient. 
Table I Characteristics of Patients Who Were Transported to a Public Emergency Department

\begin{tabular}{|c|c|c|c|}
\hline & $\begin{array}{l}\text { Matched with VEMD Record } \\
\qquad N=1,460,285\end{array}$ & $\begin{array}{l}\text { Not Matched with VEMD Record } \\
\qquad N=292,983\end{array}$ & $\begin{array}{c}\text { Standardised } \\
\text { Difference }\end{array}$ \\
\hline Age (years), mean (SD) & $55.6(25.7)$ & $49.0(26.0)$ & 0.25 \\
\hline Male sex, \% & 47.3 & 49.5 & 0.04 \\
\hline Year, (\%) & & & 0.04 \\
\hline 2015 & 20.2 & 21.6 & \\
\hline 2016 & 20.5 & 20.6 & \\
\hline 2017 & 22.1 & 21.6 & \\
\hline 2018 & 24.1 & 23.6 & \\
\hline 2019 (to June 30) & 13.1 & 12.6 & \\
\hline Time of ambulance case, $\%$ & & & 0.05 \\
\hline Day shift (7am-5pm) & 51.9 & 49.6 & \\
\hline Night shift (5pm-7am) & 48.1 & 50.4 & \\
\hline Attending crew type, \% & & & 0.06 \\
\hline Emergency ambulance & 94.6 & 95.9 & \\
\hline Non-emergency patient transport & 5.4 & 4.1 & \\
\hline Case nature, \% & & & 0.19 \\
\hline Medical & 68.8 & 60.2 & \\
\hline Trauma & 18.6 & 21.9 & \\
\hline Other & 12.6 & 17.9 & \\
\hline Paramedic treatment provided, \% & 58.9 & 54.8 & 0.08 \\
\hline Paramedic diagnosis, \% & & & 0.25 \\
\hline Pain & 19.1 & 16.4 & \\
\hline Neurological condition & 13.0 & 12.0 & \\
\hline Musculoskeletal issue/traumatic injury & 10.7 & 12.5 & \\
\hline Cardiac condition & 10.5 & 8.5 & \\
\hline Social/behavioural/mental health issue & 9.3 & 11.6 & \\
\hline Other medical condition & 9.2 & 10.6 & \\
\hline Respiratory condition & 7.8 & 5.4 & \\
\hline Infection/sepsis & 7.6 & 6.0 & \\
\hline Gastrointestinal/genitourinary condition & 5.4 & 4.7 & \\
\hline Alcohol/drug related & 3.6 & 6.4 & \\
\hline Cardiac arrest & 0.2 & 0.4 & \\
\hline No problem identified & 0.9 & 1.2 & \\
\hline Missing/unknown & 2.6 & 4.5 & \\
\hline
\end{tabular}

Note: Proportions may not add to $100 \%$ due to rounding. 
ambulance to one of the 39 public EDs that report to the VEMD, and $83.3 \%$ matched with a VEMD record. More than half $(55.3 \%)$ were admitted to hospital within 48 hours of the emergency call. Among the 216,092 patients who were referred to an alternative health service or provided with self-care advice via secondary triage, $28.3 \%$ matched with a VEMD record within 48 hours of the emergency call.

When considering patients who were transported to one of the 39 hospitals that report to the VEMD, only small differences were observed between matched and unmatched patients (Table 1). No differences were observed for sex, year, time of day or attending crew type. The data elements with the largest standardised differences between groups were patient age $(0.25)$ and paramedic diagnosis (0.25). Matched patients were older (mean \pm standard deviation: $55.6 \pm 25.7$ vs $49.0 \pm 26.0$ years) and were more likely to have a paramedic-suspected cardiac, respiratory, neurological, or gastrointestinal/genitourinary condition, suspected infection/sepsis, or pain.

\section{Discussion}

This study describes the methodology used in Victoria, Australia to link a large dataset of consecutive calls for an emergency ambulance over a 4.5-year period with hospital and death index data. Our results show that approximately $83 \%$ of patients who were transported by ambulance to a public ED matched with a VEMD record. Only small differences were observed between matched and unmatched patients.

The match rate in our study is comparable to other similar projects conducted in Australia and the United Kingdom which achieved linkage rates of between $83 \%$ and $94 \% .^{2,3,5-7,11}$ Nevertheless, in order to further increase the linkage rate, the algorithm for future linkages of this nature in Victoria could be optimised. For instance, a similar linkage conducted in New South Wales, Australia used two linkage passes to achieve a match rate of $92.4 \%$ between ambulance and administrative datasets. ${ }^{11}$ The first pass involved a deterministic linkage based on a unique Statistical Linkage Key. This was followed by a second pass which involved a probabilistic linkage of the remaining unmatched ambulance patients.

Importantly, raw health record data is generally accepted to be of lower quality and accuracy than data collected specifically for research purposes, given differing priorities at the time of data collection. ${ }^{12}$ This is particularly true in the prehospital setting where paramedics may encounter any number of challenges on scene which may hinder accurate data collection. Such data quality limitations will inherently limit the linkage rate which is achievable between ambulance and administrative datasets.

We observed relatively small differences between matched and un-matched patients, indicating a low level of bias in our dataset. ${ }^{13}$ We suggest future analysis of matched patients should acknowledge the linkage error rate. However, our findings suggest that results are likely to be generalisable to the broader ambulance population.

This linked dataset will be an invaluable resource in Victoria and will facilitate a large body of research into the patient journey across a range of clinical cohorts. It is anticipated that the dataset will be used to evaluate patient outcomes following ambulance care, including the outcomes of patients who are discharged at the scene or referred to an alternative health service via secondary triage. It will also enable evaluation of the influence of prehospital triage and management on patient outcomes. We anticipate that the linkage will be performed on an annual basis such that additional years of data can be included in the dataset.

\section{Acknowledgments}

The authors would like to acknowledge the Victorian Department of Health as the source of VAED (Victorian Admitted Episodes Dataset) and VEMD (Victorian Emergency Minimum Dataset) data for this study, the Victorian Department of Justice and Community Safety as the source of Victorian Death Index data and the Centre for Victorian Data Linkage (Victorian Department of Health) for the provision of data linkage. Emily Andrew and Shelley Cox are co-first authors for this study.

\section{Disclosure}

EA is supported by a National Health and Medical Research Council (NHRMC) Postgraduate Scholarship (\#2003449). All other authors report no conflicts of interest.

\section{References}

1. Jutte DP, Roos LL, Brownell MD. Administrative record linkage as a tool for public health research. Annu Rev Public Health. 2011;32 (1):91-108. doi:10.1146/annurev-publhealth-031210-100700

2. Carroll T, Muecke S, Simpson J, Irvine K, Jenkins A. Quantification of NSW ambulance record linkages with multiple external datasets. Prehospital Em Care. 2015;19(4):504-515. doi:10.3109/ 10903127.2015.1025154 
3. Coster J, O'Cathain A, Jacques R, et al. Outcomes for Patients Who Contact the Emergency Ambulance Service and Are Not Transported to the Emergency department: a Data Linkage Study. Prehospital Em Care. 2019;23(4):566-577. doi:10.1080/10903127.2018.1549628

4. Eastwood K, Smith K, Morgans A, Stoelwinder J. Appropriateness of cases presenting in the emergency department following ambulance service secondary telephone triage: a retrospective cohort study. $B M J$ Open. 2017;7(10):e016845. doi:10.1136/bmjopen-2017-016845

5. Crilly JL, O'Dwyer JA, O'Dwyer MA, et al. Linking ambulance, emergency department and hospital admissions data: understanding the emergency journey. Med J Australia. 2011;194(4):S34-37. doi:10.5694/j.1326-5377.2011.tb02941.x

6. Turner J, Siriwardena AN, Coster J, et al. Programme Grants for Applied Research. Developing new ways of measuring the quality and impact of ambulance service care: the PhOEBE mixed-methods research programme. Southampton (UK). NIHR J Lib. 2019;2:458.

7. Clark SJ, Halter M, Porter A, et al. Using deterministic record linkage to link ambulance and emergency department data: is it possible without patient identifiers? A case study from the UK. Int J Population Data Sci. 2019;4(1):1104. doi:10.23889/ijpds. v4i1.1104
8. Nehme Z, Bernard S, Cameron $\mathrm{P}$, et al. Using a cardiac arrest registry to measure the quality of emergency medical service care. Circ Cardiovasc Qual Outcomes. 2015;8(1):56-66. doi:10.1161/ CIRCOUTCOMES.114.001185

9. Cameron PA, Gabbe BJ, McNeil JJ, et al. The trauma registry as a statewide quality improvement tool. J Trauma Acute Care Surg. 2005;59(6):1469-1476. doi:10.1097/01.ta.0000198350.15936.a1

10. Cohen J. Statistical Power Analysis for the Behavioural Sciences. 2nd ed. New York: Academic press; 1988.

11. Cone DC, Irvine KA, Middleton PM. The Methodology of the Australian Prehospital Outcomes Study of Longitudinal Epidemiology (APOStLE) Project. Prehospital Em Care. 2012;16 (4):505-512. doi:10.3109/10903127.2012.689929

12. Weiskopf NG, Weng C. Methods and dimensions of electronic health record data quality assessment: enabling reuse for clinical research. J Am Med Informatics Assoc. 2013;20(1):144-151. doi:10.1136/ amiajnl-2011-000681

13. Harron KL, Doidge JC, Knight $\mathrm{HE}$, et al. A guide to evaluating linkage quality for the analysis of linked data. Int J Epidemiol. 2017;46(5):1699-1710. doi:10.1093/ije/dyx177

\section{Publish your work in this journal}

The International Journal of General Medicine is an international, peer-reviewed open-access journal that focuses on general and internal medicine, pathogenesis, epidemiology, diagnosis, monitoring and treatment protocols. The journal is characterized by the rapid reporting of reviews, original research and clinical studies across all disease areas. The manuscript management system is completely online and includes a very quick and fair peer-review system, which is all easy to use. Visit http://www.dovepress.com/ testimonials.php to read real quotes from published authors. 